\title{
Discrimination of JNK3 bound small molecules by saturation transfer difference NMR experiments
}

\author{
Jongsoo Lim, and Hee-Chul Ahn ${ }^{*}$ \\ Department of Pharmacy, College of Pharmacy, Dongguk University, Gyeonggi 410-820, Korea \\ (Received May 12, 2012; Revised May 24, 2012; Accepted June 08, 2012)
}

\begin{abstract}
The small molecule binding to the c-Jun N-terminal kinase 3 (JNK3) was examined by the measurements of saturation transfer difference (STD) NMR experiments. The STD NMR experiment of ATP added to JNK3 clearly showed the binding of the nucleotide to the kinase. The STD NMR spectrum of dNTPs added to JNK3 discriminated the kinase-bound nucleotide from the unbound ones. After the five-fold addition of ATP to the dNTPs and JNK3 mixture, only signals of the cognate substrate of JNK3, ATP, were observed from the STD NMR experiment. These results signify that by the STD NMR the small molecules bound to JNK3 can be discriminated from the pool of the unbound molecules. Furthermore the binding mode of the small molecule to JNK3 can be determined by the competition experiments with ATP.
\end{abstract}

Keywords: JNK3, NMR, Protein-ligand interaction, saturation transfer difference

\section{INTRODUCTION}

The c-Jun N-terminal kinases (JNKs) are members of the mitogen activated protein (MAP)

kinase family, and regulate signal transduction induced by environmental stress. MAP kinases, which include the extracellular signal regulated kinases (ERKs), p38 MAP kinases, and JNKs, are serine/threonine kinases, and are activated through phosphorylation by specific kinases upstream of

Department of Pharmacy College of Pharmacy, Dongguk University 814-9 Siksa-dong, Ilsandong-gu, Goyang, Geonggi 410-820, Korea

Tel: +82.31.961.5221 Fax:+82.31.961.5206 email: hcahn@dongguk.edu Journal of the Korean Magnetic Resonance Society 2012 June; 16(1): 67-77 http://dx.doi.org/10.6564/JKMRS.2012.16.1.067 
MAP kinases. ${ }^{1-2}$ Activated MAP kinases phosphorylate various substrates, such as transcriptional factors.

Three members of JNK kinases, JNK1, JNK2, and JNK3, have been identified and each has several different isomers. ${ }^{3}$ The transcription factor c-Jun, ${ }^{4}$ ATF- $2,{ }^{5}$ NFAT4,${ }^{6}$ and $\mathrm{p} 53,{ }^{7}$ are activated through phosphorylation by JNKs with different specificities. While JNK1 and JNK2 are widely expressed in a variety of tissues, JNK3 is mainly expressed in the brain and to a lesser extent in the heart and testis. ${ }^{3,8}$ The JNK3 signaling pathway has been reported as a critical component in the pathgenesis of glutamate neurotoxicity by the knockout experiment in mice. ${ }^{9}$ Thus, JNK3 could be a therapeutic target to prevent neurodegenerative diseases including Alzheimer's and Parkinson's diseases. Efforts aimed at developing chemical inhibitors of JNK pathway are reviewed in ref. $10{ }^{10}$

Here, we show the discrimination of JNK3 bound small molecules from a pool of compounds by using saturation transfer difference (STD) NMR spectroscopy. ${ }^{11-12}$ STD NMR experiment clearly showed the binding of ATP to JNK3. dNTPs were added JNK3 sample and STD spectrum of the mixture showed that dATP is capable of binding to the kinase. The signals of dATP were abolished when STD spectrum was recorded after the addition of ATP to JNK3-dNTP mixture. These results verified that STD NMR experiments can be used to screen small molecules which bind to JNK3 and that the binding mode of small molecule to JNK3 can be determined. 


\section{EXPERIMENTAL}

\section{Cloning, expression and purification of JNK3}

A DNA fragment encoding the kinase domain of human JNK3 spanning from S40 to E402 was amplified by PCR and cloned into the NcoI and BamH1 sites of pET15b vector. The recombinant protein was expressed in Escherichia coli BL21(DE3). Purification of JNK3 were accomplished by ion-exchange and gel filtration chromatography.

\section{NMR Spectroscopy}

The purified JNK3 was concentrated up to $10 \mathrm{mg} / \mathrm{ml}$ in $20 \mathrm{mM}$ sodium phosphate buffer, $\mathrm{pH}$ 7.2, containing $100 \mathrm{mM} \mathrm{NaCl}$ and $2 \mathrm{mM}$ DTT. 200 M ATP was dissolved in the same buffer. dNTP mixtures were dissolved in the same buffer to the final concentration of 200 M, each. All NMR experiments were carried out at $25^{\circ} \mathrm{C}$ on Agilent Unity 600 spectrometers equipped with z-axis gradient unit. One-dimensional ${ }^{1} \mathrm{H}$ spectra for ATP and dNTP mixture were respectively recorded with solvent suppression by Watergate. After the addition of JNK3 up to the final concentration of 4 M, 1D ${ }^{1} \mathrm{H}$ measurements as well as STD experiments of the chemical and protein mixtures were conducted. For STD measurement, on-resonance saturation was applied at $0 \mathrm{ppm}$ and off-resonance saturation was applied at $30 \mathrm{ppm}$. Finally, the final concentration of $1 \mathrm{mM}$ ATP was added to JNK3dNTP mixture and STD experiment was carried out. 


\section{RESULTS AND DISCUSSION}

\section{Binding of ATP to JNK3}

To detect the binding of ATP to JNK3, we employed STD NMR experiments. First, $1 \mathrm{D}{ }^{1} \mathrm{H}$ NMR spectrum of ATP was recored (Figure 1A). Since the NMR spectrum was recorded in aqueous buffer, the broad water signal was found at $4.8 \mathrm{ppm}$ even though Watergate solvent suppression was applied. Some additional peaks were also found in the spectrum, which might be from the ingredient in the buffer. All ${ }^{1} \mathrm{H}$ signals of ATP were identified and indicated by asterisks in Figure 1A. Those signals were assigned to two base protons, anomeric (1' position of ribose), 2', 3', 4', and two 5' protons, from low to high field, respectively.

${ }^{1} \mathrm{H}$ NMR spectrum of JNK3-ATP complex showed the signals from ATP and the additives as well as impurities in protein solution. Since JNK3 kinase domain used in this experiment consists of 363 amino acids and its concentration is quite low, $4 \mu \mathrm{M}$, ${ }^{1} \mathrm{H}$ signals from JNK3 might be too broad and weak to be detected. A separate 1D ${ }^{1} \mathrm{H}$ spectrum of JNK3 alone was recorded and the result showed only signals from the impurities as shown in Figure 1B. Free ATP molecules were dominant in the JNK3-ATP sample, thus, the signals from ATP were the same to those of ATP alone (Figure 1B). 
In STD NMR spectra of the JNK3-ATP mixture, two ${ }^{1} \mathrm{H}$ signals of ATP, one from the adenine base and the other from the ribose, were clearly detected, implicating that ATP binds to JNK3. The resonances from the additives and impurities still remained around 2.5-3.5 ppm even though the signals were attenuated during the STD experiments, since those are about 20 fold excess to ATP.

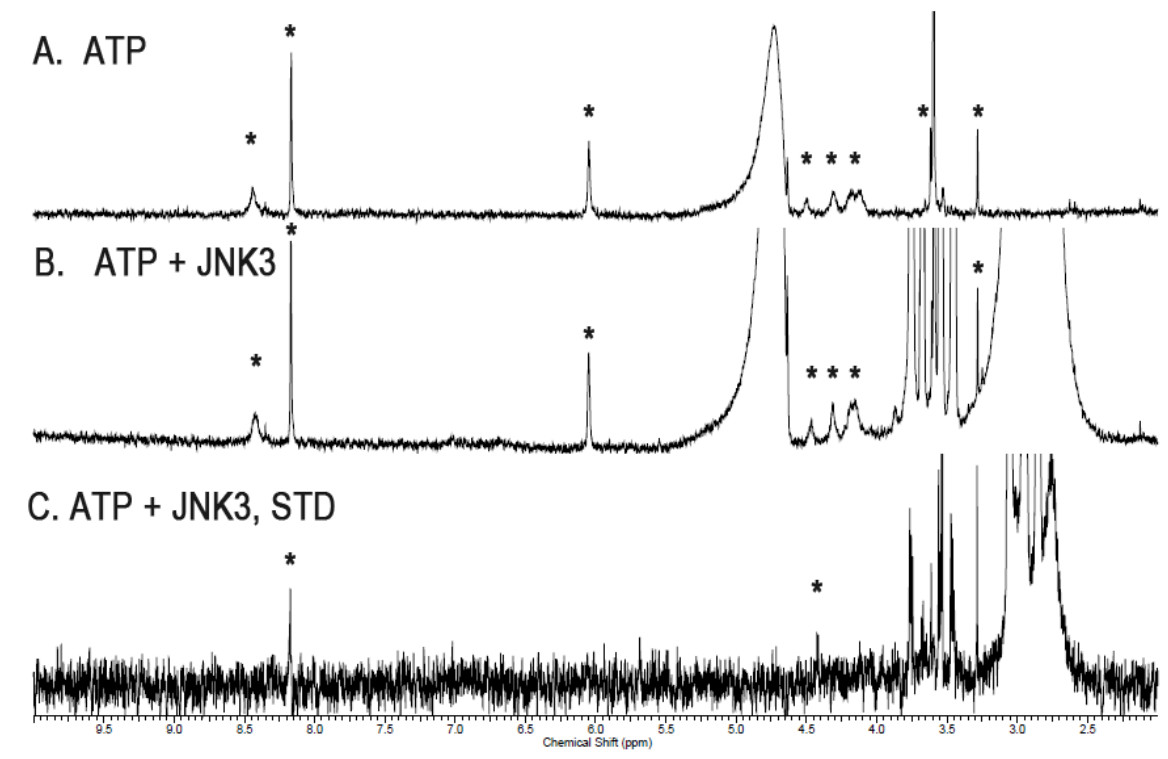

Figure 1. Binding of ATP to JNK3. (A) ${ }^{1} \mathrm{H}$ NMR spectrum of ATP in aqueous buffer. (B) ${ }^{1} \mathrm{H}$ NMR spectrum of JNK3-ATP mixture. The concentrations of JNK3 and ATP were $4 \mathrm{mM}$ and $200 \mathrm{mM}$, respectively. (C) STD NMR spectrum of JNK3-ATP mixture. Throughout the figure, ${ }^{1} \mathrm{H}$ signals of ATP were asterisked.

\section{Discrimination of JNK3 bound molecules by STD NMR}


To determine whether STD NMR measurement discriminate small molecules bound to JNK3 from a pool of several compounds, we designed simple experiments. We supposed that dATP which resembles ATP might bind to JNK3 in ATP binding pocket and other dNTPs might not. Based on this hypothesis, ${ }^{1} \mathrm{H}$ NMR spectrum of dNTPs consisting of dATP, dGTP, dCTP, and dTTP in one NMR tube was recorded. ${ }^{1} \mathrm{H}$ NMR measurement and STD NMR experiment of JNK3-dNTP mixture were followed.

Proton signals from the bases and anomeric positions of each dNTPs were well resolved in ${ }^{1} \mathrm{H}$ NMR spectrum of JNK3-dNTP mixture (Figure 2A). Signals from ribose ring were excluded in the analysis because those were crowded and overlapped with impurities. However, STD NMR results clearly discriminated the JNK3 bound molecule from unbound ones. The individual ${ }^{1} \mathrm{H}$ NMR measurements of dNTPs enabled the resonance assignments of STD NMR signals in Figure 2B. The resonances at 8.4 and $8.2 \mathrm{ppm}$ correspond to those of protons from adenine base and the signal at 6.4 $\mathrm{ppm}$ is that of anomeric proton of dATP. The proton signal at $6.0 \mathrm{ppm}$ is not clear due to the signal overlap of dNTPs. From this experiment, we verified that at least dATP can bind to JNK3 and that JNK3 bound molecules can be selectively detected from the mixture of several compounds. Indeed several kinases has been reported to have considerable binding affinities to dATP. ${ }^{13-14}$ 


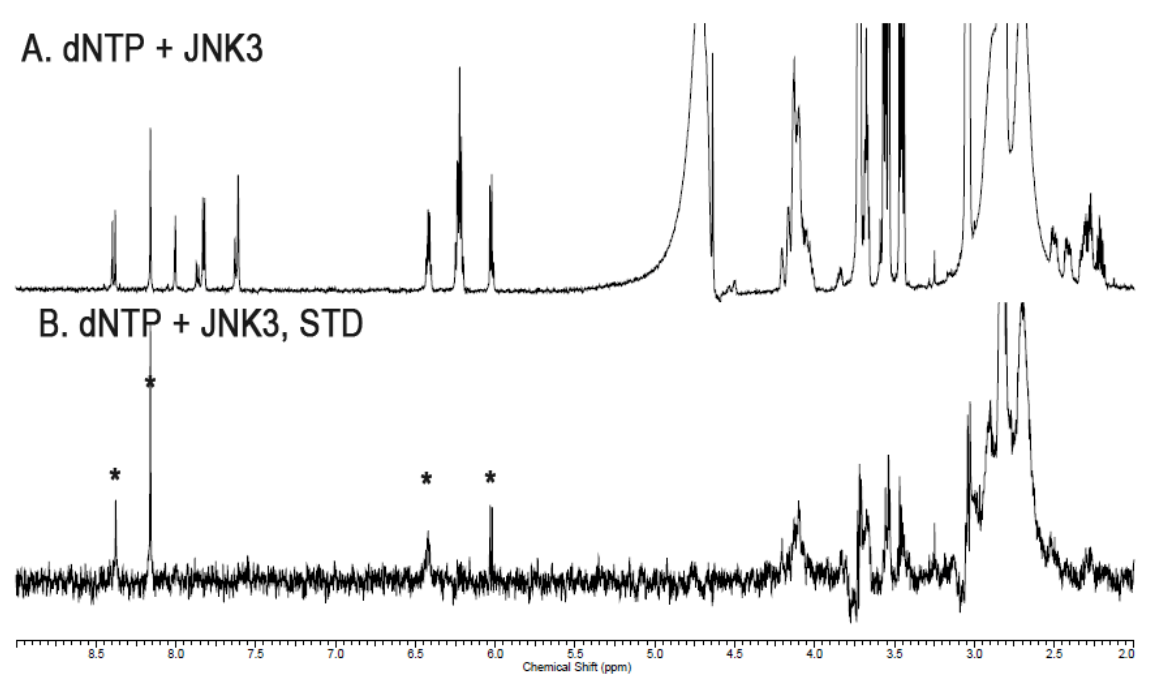

Figure 2. Discrimination of JNK3 bound molecules by STD NMR. (A) ${ }^{1} \mathrm{H}$ NMR spectrum of JNK3dNTP mixtures. (B) ${ }^{1} \mathrm{H}$ signals of dATP were selectively identified from STD NMR experiments. Asterisked peaks were STD signals of JNK3 bound molecules and three of them were assigned to protons of dATP.

\section{Competition experiment between JNK3 bound molecules by STD NMR}

ATP was added to JNK3-dNTP mixture and STD NMR spectrum was recorded. In ${ }^{1} \mathrm{H}$ NMR spectrum of JNK3-dNTP-ATP mixture showed individual resonances of nucleotides with higher intensities of ATP signals because the amount of added ATP was five-fold excess to those of dNTPs (Figure 3A). In STD NMR spectrum of the sample, only signals of ATP were detected (Figure 3B). This result means that the cognate substrate of JNK3, ATP, replaced dATP, the non-cognate JNK3 binder, at ATP binding pocket in JNK3. 


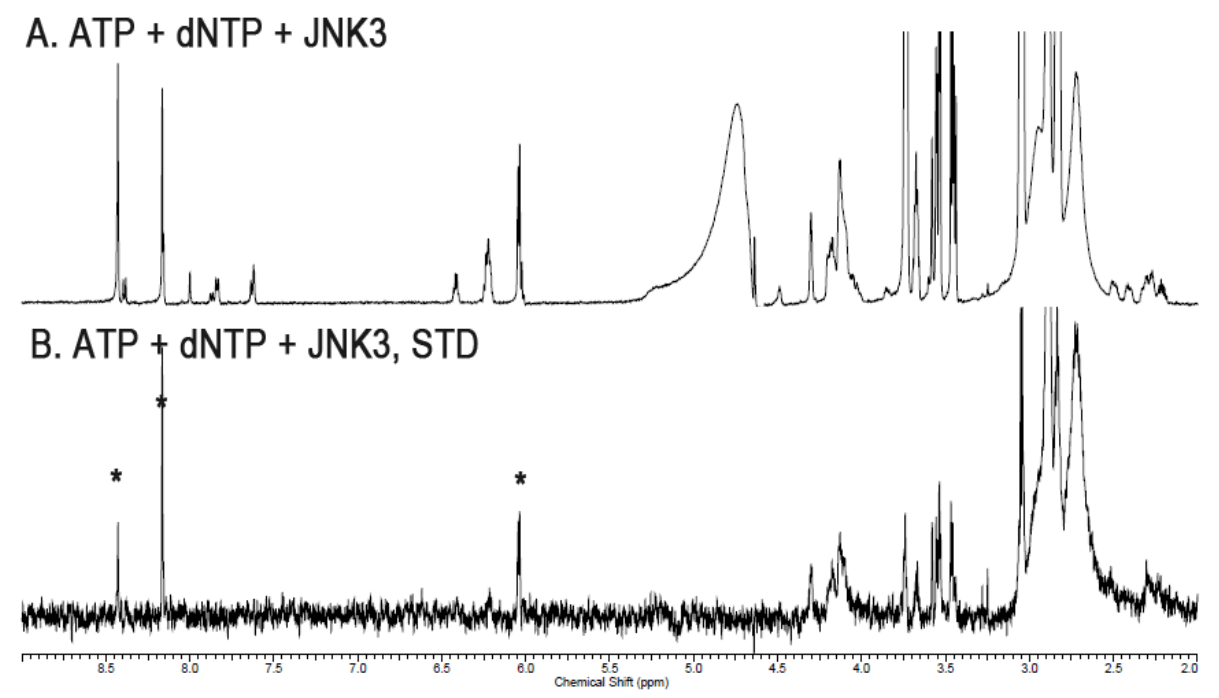

Figure 3. Competition experiment between JNK3 bound molecules by STD NMR. (A) ${ }^{1} \mathrm{H}$ NMR spectrum of JNK3-dNTP-ATP mixture. (B) STD NMR spectrum of JNK3-dNTP-ATP mixture. In STD NMR spectrum, the resonances of ATP were remained and asterisked.

\section{Implication in small molecule screening against JNK3}

NMR spectroscopy is not only one of the most powerful tools in the identification of chemicals but also a highly versatile screening method in drug discovery. Since NMR signals of compounds from free- and protein-bound forms are quite different, it is easy and straight-forward to distinguish certain chemical(s) which can bind to the target protein from a pool of chemicals. STD NMR spectroscopy is a powerful method for studying protein-ligand interactions in solution. The STD NMR method is capable of identifying the binding epitope of a ligand when it bound to its receptor 
protein. ${ }^{15}$ Ligand protons that are in close contact with the receptor protein receive a higher degree of saturation, and as a result stronger STD NMR signals can be observed. Protons that are either less or not involved in the binding process reveal no STD NMR signals.

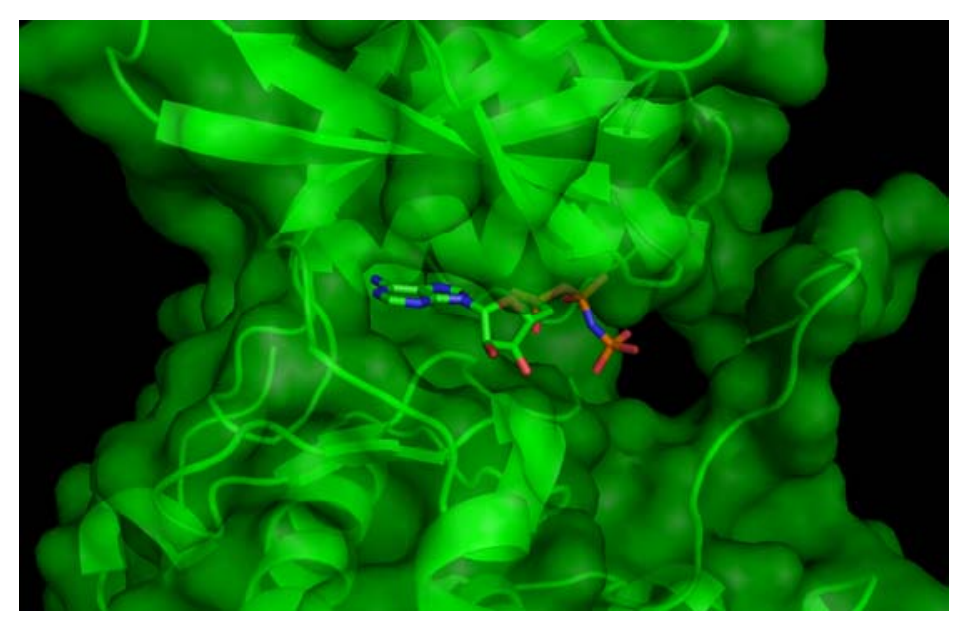

Figure 4. Active site structure of JNK3 (PDB 1JNK, ref 16). ATP derivative is complexed on the active site of JNK3.

In this work, versatility of STD NMR spectroscopy was evaluated to detect the binding of small molecules to JNK3. We hypothesized that dATP might bind to JNK3 since its structure is very similar to ATP. We prepared the mixture of four dNTPs in one NMR tube and measured STD NMR spectra with JNK3. As expected dATP showed to have significant JNK3 binding affinity while the rest three nucleotides did not. Indeed several kinases has been reported to have considerable binding 
76 Discrimination of JNK3 bound small molecules by NMR experiments

affinities to dATP, and dGTP with much less extent. ${ }^{13-14}$ Thus, we successfully discriminated JNK3 binder, dATP, from a pool of dNTP mixtures.

Since the addition of ATP to JNK3-dATP complex abolished the dATP resonances in STD NMR spectrum, we could conclude that ATP and dATP compete at the active site of the kinase (Figure 4). Thus, in this work we verified that STD NMR method could be very effective way of screening small molecules targeted JNK3 and whether the binding site is the active site or not could be estimated by the competitive STD NMR experiment. Based on these experiments here, our laboratory is pursuing screening small molecules for discovery of inhibitor targeted on JNK3.

\section{Acknowledgment}

This work was supported by the GRRC program of Gyeonggi province. [(GRRCDONGGUK2011-A01), Study of control of viral diseases].

\section{REFERENCES}

1. M. H. Cobb, E. J. Goldsmith, J. Biol. Chem. 270, 14843 (1995).

2. A. Minden, M. Karin, Biochem. Biophys. Acta 1333, F85 (1997).

3. S. Gupta, T. Barrett, A. J. Whitmarsh, J. Cavanagh, H. K. Sluss, B. Dérijard, R. J. Davis, EMBO J. 15, 2760 (1996).

4. Y. Zhang, L. Zhou, C. A. Miller, Proc. Natl Acad. Sci. USA 95, 2586 (1998).

5. H. van Dam, D. Wilhelm, I. Herr, A. Stetfen, P. Herrlich, P. Anglel, EMBO J. 14, 1798 (1995).

6. C. W. Chow, M. Rincon, J. Cavanagh, M. Dickens, R. J. Davis, R. J. Science 278, 1638 (1997).

7. D. M. Milne, L. E. Campbell, D. G. Campbell, D. W. Meek, J. Biol. Chem. 270, 5511 (1995).

8. A. A. Mohit, J. H. Martin, C. A. Miller, C. A. Neuron 14, 67 (1995). 
9. D. D. Yang, C. Y. Kuan, A. J. Whitmarsh, M. Rincón, T. S. Zheng, R. J. Davis, P. Rakic, R. A. Flavell, Nature 389, 865 (1997).

10. T. Borsello, F. Gianluigi, Curr. Pharm. Des. 13, 1875 (2007).

11. M. Mayer, B. Meyer, Angew. Chem. Int. Ed. 38, 1784 (1999).

12. B. Meyer, T. Peters, Angew. Chem. Int. Ed. 42, 864 (2003).

13. N. D. Richert, D. L. Blithe, I. Pastan, J. Biol. Chem. 257, 7143 (1982).

14. L. -S. Lee, Y. -C. Cheng, Biochemistry 15, 3686 (1976).

15. T. Haselhorst, A. C. Lamerz, M. Itzstein, Methods Mol. Biol. 534, 375 (2009).

16. X. Xie, Y. Gu, T. Fox, J. T. Coll, M. A. Fleming, W. Markland, P. R. Caron, K. P. Wilson, M. SS. Su, Structure 6, 983 (1998). 